\title{
Study on the Clinical Efficacy of Bailing Tablets Combined with Strengthening the Spleen and Kidney in the Treatment of Nephropathy
}

WENZHONG XIONG*, JING ZHOU, ZHAOXIA XIAAND JING TANG

Department of Endocrinology and Metabolism, Luzhou People's Hospital, Luzhou 646000, Sichuan, China

Xiong et al.: Efficacy of Bailing Tablets in the Treatment of Patients with Nephropathy

\begin{abstract}
To observe and study the clinical efficacy of Bailing tablets combined with the method of strengthening the spleen, benefiting the kidney, removing turbidity and removing blood stasis in the treatment of patients with gouty nephropathy, and to provide a reliable theoretical basis and clinical basis. A total of 120 patients with primary gouty nephropathy diagnosed in our hospital from December 2018 to December 2020 were divided into intervention group and control group (randomized). The control group was treated with Bailing tablets alone, while the intervention group was treated with Bailing tablets combined with the method of strengthening the spleen, benefiting the kidney, removing turbidity and removing blood stasis. Clinical efficacy, syndrome efficacy, renal function, urine protein, immune status, psychological status, etc., were compared between the two groups after treatment. The intervention group significantly improved the clinical overall response rate $(93.3 \%$ vs. $81.6 \%, p<0.05)$, with a post-treatment creatinine of $(127.1 \pm 23.1) \mu \mathrm{mol} / \mathrm{l}$ in the intervention group compared to $(140.3 \pm 37.3) \mu \mathrm{mol} / \mathrm{l}$ in the control group $(p<0.05)$. The cluster of differentiation 4/cluster of differentiation 8 in the treatment group was $(1.23 \pm 0.17)$, which was significantly lower than that $(1.89 \pm 0.18)$ in the control group $(p<0.05)$. Adverse effects were generally similar between the two groups $(p>0.05)$. The treatment of gouty nephropathy using Bailing tablets combined with the method of strengthening the spleen, benefiting the kidney, removing turbidity and removing blood stasis is clinically important for improving renal function, reducing the occurrence of adverse effects and improving systemic immunity, and is a safe and effective drug for the treatment of gouty nephropathy.
\end{abstract}

Key words: Bailing tablets, traditional Chinese medicine, blood stasis, gouty nephropathy, cluster of differentiation cells

Gout refers to a variety of metabolic diseases characterized by the deposition of monosodium urate crystals in tissues. On the renal side, gout manifests as acute or chronic gouty nephropathy and urolithiasis ${ }^{[1]}$. Studies have shown that elevated serum uric acid independently predicts the development of Chronic Kidney Disease (CKD) and gout is present in one-third of patients with chronic renal insufficiency. Bardin et al. have reported that one-third of gout patients have hyperechoic ear cords (consistent with crystal deposition), which is associated with an increased risk of hypertension and renal insufficiency ${ }^{[2]}$. Therefore, early and aggressive intervention in gouty nephropathy can prevent the gradual deterioration of the condition from progressing to renal failure and greatly improve

*Address for correspondence

E-mail: xiong123wenzhong@163.com the quality of life of patients.

At present, conventional treatment drugs include nonsteroidal anti-inflammatory drugs, corticosteroids, allopurinol, etc., and long-term treatment is required for their recurrent attacks. Since each drug has its own side effects and limitations, careful selection of the drug to be used should always be made. Traditional Chinese Medicines (TCMs) have long been used to treat gout and are characteristically useful for the management of gouty nephropathy. At present, a large number of clinical trials of TCMs have been carried out and widely used in clinical practice for the treatment of gout ${ }^{[3]}$. At this stage, the effect of Bailing tablets combined with the method of strengthening the spleen, benefiting the 
kidney, removing turbidity and removing blood stasis in the treatment of gouty nephropathy is temporarily unclear, and this study is intended to explore the clinical efficacy of Bailing tablets combined with the method of strengthening the spleen, benefiting the kidney, removing turbidity and removing blood stasis in the treatment of gouty nephropathy, thereby providing a scientific basis for subsequent treatment.

\section{MATERIALS AND METHODS}

\section{Study objects:}

A total of 120 patients with primary gouty nephropathy from December 2018 to December 2020 in our hospital were selected and after obtaining the informed consent of the patients in advance, the patients were randomly divided into the intervention group and the control group. The control group was treated with Bailing tablets alone, the intervention group was treated with Bailing tablets combined with the method of strengthening the spleen, benefiting the kidney, removing turbidity and removing blood stasis, and those patients who cannot communicate with severe mental illness are not included.

\section{Methods:}

All patients were treated with the following; such as guaranteeing a low purine diet, avoiding the use of irritating foods, encouraging increased water intake, guaranteeing urine output and encouraging the use of basic foods to alkalinize urine and maintaining mood. The control group was treated with Bailing tablets alone, while the intervention group was treated with Bailing tablets + The method of strengthening the spleen, benefiting the kidney, removing turbidity and removing blood stasis.

\section{Efficacy measures:}

It is made according to the "Protocol for the Diagnosis, Syndrome Differentiation and Evaluation of Uric Acid Nephropathy (Trial protocol) ${ }^{[4]}$.

\section{Statistical methods:}

Data analysis was performed using Statistical Package for the Social Sciences (SPSS) 24 statistics and the measurement data were analyzed by t-test and expressed as mean \pm Standard Deviation (SD) $(\mathrm{x} \pm \mathrm{s})$; comparisons between groups were performed by two independent samples t-test and comparisons of count data [n (\%)] were performed by $\chi^{2}$ test when $p<0.05$ were considered statistically significant.

\section{RESULTS AND DISCUSSION}

Baseline data was given where 60 patients in the intervention group were of age (35-72 y) and 28 were female. In the control group, 60 patients were of age (34-75 y) and 29 were female (Table 1).

Clinical efficacy between the two groups was compared. According to the clinical symptoms, serum uric acid levels, urinary microalbumin, serum creatinine levels were divided into clinical control, producing effect, effective, ineffective; the intervention group was more effective than the control group $(\mathrm{p}<0.05)$, as detailed in Table 2.

Syndrome efficacy between the two groups was compared. According to TCM clinical symptoms, signs and syndrome scores were divided into clinical control, producing effect, effective and ineffective; the syndrome efficacy in the intervention group was better than that in the control group $(\mathrm{p}<0.05)$, as detailed in Table 3.

Renal function between the two groups was compared. When comparing the renal function indicators such as creatinine, urea nitrogen and uric acid between the two groups before and after treatment, there was no obvious difference in each indicator of renal function between the two groups, which was evaluated again after treatment. It can be seen that the recovery of renal function in the intervention group was more obvious than that in the control group $(\mathrm{p}<0.05)$, as detailed in Table 4.

Beta-2 Microglobulin ( $\left.\beta_{2}-\mathrm{MG}\right)$ and urine protein levels before and after treatment in the two groups were compared. By comparing $\beta_{2}-\mathrm{MG}, 24 \mathrm{~h}$ urine protein of the two groups of patients before and after treatment, it can be seen that the urine protein of the kidney in the intervention group after treatment was lower, as detailed in Table 5.

Peripheral blood lymphocyte subsets between the two groups were compared. By comparing the lymphocyte subsets in peripheral blood before and after treatment between the two groups, we can see that there was no difference between the two groups before treatment, while in the intervention group, the Cluster of Differentiation $\mathrm{CD}^{+} / \mathrm{CD}^{+}$were higher, while $\mathrm{CD}^{+} /$ $\mathrm{CD}^{+}$were lower after treatment, as detailed in Table 6 .

Adverse reactions between the two groups were compared. Adverse effects such as nausea and vomiting, diarrhea, skin rash, and loss of appetite were compared between the two groups, and the incidence of total adverse effects was not significantly different between the two groups ${ }^{[5]}$ (Table 7). 
TABLE 1: BASELINE DATA OF THE TWO GROUPS

\begin{tabular}{|c|c|c|c|c|}
\hline & $\begin{array}{l}\text { Intervention group } \\
\qquad(n=60)\end{array}$ & Control group $(n=60)$ & $t / \chi^{2}$ & $\mathrm{p}$ \\
\hline Age & $53.1 \pm 16.1$ & $52.5 \pm 17.9$ & 0.20 & 0.83 \\
\hline Gender (female) & $28(\%)$ & $29(\%)$ & 0.03 & 0.86 \\
\hline \multicolumn{5}{|l|}{ Complication } \\
\hline Hypertension & $32(53.3 \%)$ & $30(50.0 \%)$ & 0.14 & 0.71 \\
\hline $\begin{array}{l}\text { Coronary Heart Disease } \\
(\mathrm{CHD})\end{array}$ & $24(40 \%)$ & $26(43.3 \%)$ & 0.30 & 0.58 \\
\hline Diabetes mellitus & $33(55.0 \%)$ & $30(50 \%)$ & 0.21 & 0.65 \\
\hline Tumor & $3(5.0 \%)$ & $2(3.3 \%)$ & 0.14 & 0.71 \\
\hline
\end{tabular}

TABLE 2: COMPARISON OF CLINICAL EFFICACY BETWEEN THE TWO GROUPS

\begin{tabular}{|c|c|c|c|c|c|}
\hline & Clinical controls & Producing effect & Effective & Ineffective & Total effective rate \\
\hline Intervention group & $18(30.0 \%)$ & $25(41.6 \%)$ & $14(23.3 \%)$ & $4(6.7 \%)$ & $56(93.3 \%)$ \\
\hline Control group & $14(23.3 \%)$ & $20(33.3 \%)$ & $15(25 \%)$ & $11(18.3 \%)$ & $49(81.6 \%)$ \\
\hline$\chi^{2}$ & & & & & 4.62 \\
\hline $\mathrm{p}$ & & & & & 0.03 \\
\hline
\end{tabular}

TABLE 3: COMPARISON OF SYNDROME EFFICACY BETWEEN THE TWO GROUPS

\begin{tabular}{|c|c|c|c|c|c|}
\hline & Clinical cure & Producing effect & Effective & Ineffective & $\begin{array}{c}\text { Overall } \\
\text { satisfaction rate }\end{array}$ \\
\hline Intervention group & $19(31.6 \%)$ & $18(30.0 \%)$ & $20(33.3 \%)$ & $3(5.0 \%)$ & $57(95.0 \%)$ \\
\hline Control group & $12(20 \%)$ & $22(36.7 \%)$ & $16(26.7 \%)$ & 10 (16.7 \%) & $50(83.3 \%)$ \\
\hline$\chi^{2}$ & & & & & 4.33 \\
\hline $\mathrm{p}$ & & & & & 0.04 \\
\hline
\end{tabular}

TABLE 4: COMPARISON OF RENAL FUNCTION BETWEEN THE TWO GROUPS

\begin{tabular}{lccccc}
\hline & & $\begin{array}{c}\text { Intervention group } \\
(\mathrm{n}=60)\end{array}$ & $\begin{array}{c}\text { Control group } \\
(\mathrm{n}=60)\end{array}$ & $\mathrm{t}$ & $\mathrm{p}$ \\
\hline \multirow{2}{*}{ Creatinine $(\mu \mathrm{mol} / \mathrm{l})$} & Before treatment & $167.1 \pm 26.1$ & $158.2 \pm 27.3$ & 1.82 & 0.07 \\
& After treatment & $127.1 \pm 23.1$ & $140.3 \pm 37.3$ & -2.22 & 0.02 \\
Urea nitrogen & Before treatment & $15.6 \pm 5.1$ & $16.1 \pm 4.4$ & -0.57 & -2.77 \\
$(\mu \mathrm{mol} / \mathrm{l})$ & After treatment & $7.4 \pm 3.6$ & $9.3 \pm 3.9$ & 0.91 & 0.01 \\
\multirow{2}{*}{ Uric acid $(\mu \mathrm{mol} / \mathrm{l})$} & Before treatment & $567.1 \pm 56.1$ & $557.6 \pm 58.2$ & -2.83 & 0.36 \\
& After treatment & $457.1 \pm 46.4$ & $483.2 \pm 56.2$ & 0.01 \\
\hline
\end{tabular}

TABLE 5: COMPARISON OF $\beta_{2}$-MG AND 24 h URINE PROTEIN LEVELS BETWEEN THE TWO GROUPS

\begin{tabular}{lccccc}
\hline & & $\begin{array}{c}\text { Intervention group } \\
(\mathrm{n}=60)\end{array}$ & $\begin{array}{c}\text { Control group } \\
(\mathrm{n}=60)\end{array}$ & $\mathrm{t}$ & $\mathrm{P}$ \\
\hline $\mathrm{B}_{2}-\mathrm{MG}(\mathrm{mg} / \mathrm{l})$ & Before treatment & $4.01 \pm 0.47$ & $4.04 \pm 0.66$ & -0.28 & 0.77 \\
& After treatment & $2.27 \pm 0.06$ & $3.00 \pm 0.07$ & -66 & 0.000 \\
$24 \mathrm{~h}$ Total protein & Before treatment & $1.26 \pm 0.08$ & $1.27 \pm 0.07$ & -0.91 & 0.36 \\
(TP) $(\mathrm{g} / 24 \mathrm{~h})$ & After treatment & $0.66 \pm 0.02$ & $0.80 \pm 0.01$ & -5.31 & 0.000 \\
\hline
\end{tabular}


TABLE 6: COMPARISON OF PERIPHERAL LYMPHOCYTE SUBSETS BETWEEN THE TWO GROUPS

\begin{tabular}{|c|c|c|c|c|c|}
\hline & & $\begin{array}{l}\text { Intervention group } \\
(\mathrm{n}=60)\end{array}$ & $\begin{array}{l}\text { Control group } \\
(n=60)\end{array}$ & $\mathrm{t}$ & $\mathrm{p}$ \\
\hline \multirow{2}{*}{$\mathrm{CD}^{+}$} & Before treatment & $59.36 \pm 3.23$ & $58.35 \pm 4.12$ & 1.49 & 0.14 \\
\hline & After treatment & $69.36 \pm 4.12$ & $59.18 \pm 4.01$ & 13.37 & 0.001 \\
\hline \multirow{2}{*}{$\mathrm{CD}^{+} / \mathrm{CD}^{+}$} & Before treatment & $25.28 \pm 2.98$ & $25.34 \pm 3.58$ & -0.1 & 0.92 \\
\hline & After treatment & $36.32 \pm 3.01$ & $29.81 \pm 4.56$ & 9.92 & 0.001 \\
\hline \multirow{2}{*}{$\mathrm{CD}^{+} / \mathrm{CD}^{+}{ }^{+}$} & Before treatment & $2.79 \pm 0.13$ & $2.75 \pm 0.13$ & 1.68 & 0.09 \\
\hline & After treatment & $1.23 \pm 0.17$ & $1.89 \pm 0.18$ & -20 & 0.0001 \\
\hline
\end{tabular}

TABLE 7: COMPARISON OF ADVERSE EFFECTS BETWEEN THE TWO GROUPS

\begin{tabular}{|c|c|c|c|c|c|}
\hline & $\begin{array}{c}\text { Nausea and } \\
\text { vomiting }\end{array}$ & Diarrhea & Skin rash & Loss of appetite & $\begin{array}{c}\text { Incidence of } \\
\text { adverse reactions }\end{array}$ \\
\hline Intervention group & $5(\%)$ & 1 (\%) & 1 (\%) & 1 (\%) & $8(13.3 \%)$ \\
\hline Control group & $4(\%)$ & $4(\%)$ & 1 (\%) & $5(\%)$ & 12 (20\%) \\
\hline$\chi^{2}$ & & & & & 0.96 \\
\hline $\mathrm{p}$ & & & & & 0.32 \\
\hline
\end{tabular}

The etiopathogenesis of gouty nephropathy is extremely complex and the specific pathogenesis of gouty nephropathy is not clear in western medicine. At present, the kidney is the main organ for urate excretion and the urate excretion fraction is determined by glomerular filtration and tubular reabsorption and secretion. Uric acid is deposited in the kidney in various segments of the renal tubules, obstructing the tubules and causing them to dilate, atrophy and even causing fibrosis and necrosis, thus leading to gouty nephropathy ${ }^{[6]}$. Studies have shown that uric acid crystals have been shown to initiate Interleukin-1 Beta (IL-1 $\beta$ )-mediated inflammation by activating the Nod-Like Receptor Protein 3 (NLRP3) inflammasome ${ }^{[7]}$. In addition, the expression of lipid metabolism was increased in gouty nephropathy, which may mediate the progression of gouty nephropathy through Phospholipases A2 (PLA2) activation, $\beta$-oxidation and activation. Shao et al. found that the fecal microbiome and metabolome of patients with gout can be characterized by the disturbance of metabolites involved in the excretion of uric acid and changes in amino acids directly responsible for purine nucleoside biosynthesis, but also by the enrichment of opportunistic pathogens and the reduction of alpha diversity ${ }^{[8]}$. Western medical treatments are mainly antiinflammatory, analgesic, uric acid lowering, nascent drugs and other treatments ${ }^{[9,10]}$. However, the high cost and inherent deficiencies of biopharmaceuticals limit the application of these drugs. The currently available first-line drugs for the treatment of gouty arthritis are associated with multiple adverse effects. Therefore, identifying alternative therapeutic strategies is particularly urgent and important. 
controlled experiments is necessary.

Bailing tablets combined with the method of strengthening the spleen, benefiting the kidney, removing turbidity and removing blood stasis can alleviate clinical symptoms, restore renal function and elevate immune function of patients with gouty nephropathy, and other effects.

\section{Author's contributions:}

Wenzhong Xiong and Jing Zhou contributed equally to this work.

\section{Conflict of interests:}

The authors declared no conflict of interest.

\section{REFERENCES}

1. Zurek M. Pathogenesis, diagnostics and therapy of gout. Vnitr Lek 2006;52(7-8):736-41.

2. Piani F, Johnson RJ. Does gouty nephropathy exist, and is it more common than we think? Kidney Int 2021;99(1):31-3.

3. Zhang J, Pan H, Xie J, Wang J, Wang R, Qiu X, et al. Serum metabolic profiling analysis of gout patients treated with traditional Chinese medicine Tongfengtai granules based on gas chromatography-mass spectrometry. Evid Based Complement Alternat Med 2020;2020: 7404983.

4. Nephrology branch of Chinese academy of traditional Chinese medicine. Diagnosis, syndrome differentiation, and efficacy assessment of uric acid nephropathy (trial protocol)\%. Shanghai J Tradit Chin Med 2008;42(1).

5. Liang N, Sun M, Sun R, Xu T, Cui L, Wang C, et al. Baseline urate level and renal function predict outcomes of urate-lowering therapy using low doses of febuxostat and benzbromarone: A prospective, randomized controlled study in a Chinese primary gout cohort. Arthritis Res Ther 2019;21(1):1-9.

6. Guo H, Fang M. Gouty nephropathy. J Pract Village Doct China 2019;11(26).

7. Zhang YZ, Sui XL, Xu YP, Gu FJ, Zhang AS, Chen JH. NLRP3 inflammasome and lipid metabolism analysis based on UPLC-Q-TOF-MS in gouty nephropathy. Int J Mol Med 2019;44(1):172-84.
8. Shao T, Shao L, Li H, Xie Z, He Z, Wen C. Combined signature of the fecal microbiome and metabolome in patients with gout. Front Microbiol 2017;8:268.

9. Chen S, Yu HZ, Fang ZZ, Yu T, Chen C, Zhong H. An overview of the progress of traditional Chinese medicine in the treatment of gouty nephropathy. J Emerg Chin Tradit Med 2020;29(10):1877-80.

10. Yao R, Geng Z, Mao X, Bao Y, Guo S, Bao L, et al. Tu-TengCao extract alleviates monosodium urate-induced acute gouty arthritis in rats by inhibiting uric acid and inflammation. Evid Based Complement Alternat Med 2020;2020.

11. Sun Y, Xi R, Fu SF, Guo JC. A review of traditional Chinese and western medicine for the treatment of gouty nephropathy. Rheumatol Arthritis 2020;8(9).

12. Liu Z, Liu W, Zhang Y. Data mining based analysis of the drug regularity of traditional Chinese medicine for the treatment of gouty nephropathy. Rheumatol Arthritis 2020;8(9).

13. Wang Hc, Liu J, Clinical study of Bailing capsule combined with allopurinol on the treatment of gouty nephropathy. Mod Drug Clin 2018;33(3).

14. Ma Z, Yang GW. Efficacy and effect on immune function of Bailing tablets in chronic glomerulonephritis. Tradit Chin Med Clin Res 2017;9(34).

15. Zhang DC, Li L, Zhao XF, Li YJ. Clinical observation of tonifying spleen and kidney, relieving turbidity and removing stasis soup combined with Shuangbai Powder for the treatment of acute gouty arthritis. J Emerg Tradit Chin Med 2014;23(10):1919-21.

16. Zheng Q, Sun LY, Zhao Q, Zhang XX, Wang Y, Jin H, et al. Efficacy and safety of Bushenxiezhuo therapy versus allopurinol in the treatment of gouty nephropathy: A Metaanalysis. Chin Gen Pract 2019;22(8):947-53.

17. Hu J, Liu Y, Liu HF. Prof. Liu Hongfang based on turbid stasis heat and toxin for the treatment of gouty nephropathy. Mod Remote Educ Chin Tradit Med 2020;5(18).

This is an open access article distributed under the terms of the Creative Commons Attribution-NonCommercial-ShareAlike 3.0 License, which allows others to remix, tweak, and build upon the work non-commercially, as long as the author is credited and the new creations are licensed under the identical terms

This article was originally published in a special issue, "Novel Therapeutic Approaches in Biomedicine and Pharmaceutical Sciences" Indian J Pharm Sci 2021:83(6) Spl Issue "130-134" 